\title{
Efecto de la disposición de los residuos resultantes del beneficiado húmedo del café sobre las características físicas y química del agua de corriente natural Effect of the disposal of coffee wet milling residues on the physical and chemical characteristics of natural flowing water
}

\author{
Ramiro Eleazar Ruiz-Nájera ${ }^{1 *}$ (D), José Alfredo Medina-Meléndez ${ }^{1}$ (D), Jesús Carmona-de la Torre ${ }^{2}$ (D), \\ Gabriel Rincón-Enríquez ${ }^{3}$ (i) Juan Manuel Sánchez-Yáñez ${ }^{4}$ (i) y Deb Raj-Aryal ${ }^{1}$ (i)

\footnotetext{
${ }^{1}$ Universidad Autónoma de Chiapas, Facultad de Ciencias Agronómicas, Campus V. Carretera Ocozocoautla-Villaflores km 81. 30470 Villaflores, Chiapas, México. * Autor para correspondencia (ruiznajerar@yahoo.com)

${ }^{2}$ Colegio de la Frontera Sur (ECOSUR), Área de Laboratorios Institucionales, Unidad San Cristóbal de las Casas. Periférico sur s/n, María Auxiliadora. 29290 San Cristóbal de las Casas, Chiapas, México.

${ }^{3}$ Centro de Investigación y Asistencia en Tecnología y Diseño del Estado de Jalisco. Camino Arenero 1227, El Bajío del Arenal. 45019 Zapopan, Jalisco, México.

${ }^{4}$ Universidad Michoacana de San Nicolás de Hidalgo. Instituto de Investigaciones Químico-Biológicas, Laboratorio de Microbiología Ambiental, edificio B-3, Ciudad Universitaria Francisco J. Mujica s/n, Felicitas del Río. 58000 Morelia, Michoacán, México.
}

\section{RESUMEN}

El beneficio húmedo del café demanda grandes cantidades de agua, además de generar mucílago y pulpa que se integran a las aguas superficiales. El objetivo de esta investigación fue analizar las propiedades físicas y químicas del agua de río Cuxtepec, río Cabañas y su principal afluente, ubicado en La Concordia, Chiapas, México. En estas aguas son descargados los subproductos derivados del beneficiado húmedo del café durante el periodo de cosecha. Los sitios de muestreo fueron arroyo San Pedro, río Cabañas, ranchería 30 de Agosto y río Cuxtepec, las muestras de agua se obtuvieron durante octubre y diciembre 2016; enero, abril y junio 2017. Se determinó $\mathrm{pH}$, conductividad eléctrica (CE), sólidos disueltos totales (SDT), sólidos suspendidos totales (SST), sólidos sedimentables (SS), demanda química de oxígeno (DQO), demanda bioquímica de oxígeno (DBO), nitrógeno total $(\mathrm{NT})$, amonio $\left(\mathrm{NH}_{4}^{+}\right)$, nitritos $\left(\mathrm{NO}_{2}^{-}\right)$, nitratos $\left(\mathrm{NO}_{3}^{-}\right)$como $\mathrm{N}$, fósforo total (PT), dureza del agua (DA), temperatura del agua (TA) y la temperatura ambiental (T. Amb). El contenido de DQO y DBO varió de un sitio a otro, con valores máximos entre 7.7 y $1.25 \mathrm{mg} \mathrm{L}^{-1}$, respectivamente. Esta baja concentración permitió altos contenidos de oxígeno al nivel de $7.99 \mathrm{mg} \mathrm{L}^{-1}$. Asimismo, la carga de SDT fue de $149.7 \mathrm{mg} \mathrm{L}^{-1} \mathrm{y} \mathrm{pH}$ de 8.9 , estas son condiciones favorables para la vida acuática. En general, los valores medidos en corrientes aguas abajo a las plantas tradicionales del procesamiento húmedo de café no presentaron niveles que indiquen riesgos para los usos del agua en actividades agrícolas, pecuarias y consumo humano en las áreas cercanas a los cuerpos de agua. Estos hallazgos plantean la necesidad de establecer un sistema de monitoreo de los contaminantes señalados en la zona cafetalera de Chiapas, para mejorar el manejo ecológico de las aguas residuales provenientes del beneficiado húmedo del café.

Palabras clave: fuentes de agua, musilago, propiedades del agua, pulpa de café.

\section{SUMMARY}

Coffee wet milling is a process that demands large quantities of water, and produces mucilage and pulp that are discharged into surface water. The objective of this study was to analyze the physical and chemical properties of the water in Cuxtepec and Cabañas rivers in the municipality of Concordia, Chiapas, Mexico. In these water masses, coffee wet milling subproducts are

Cita recomendada:

Ruiz-Nájera, R. E., Medina-Meléndez, J. A., Carmona-de la Torre, J., Rincón-Enríquez, G., Sánchez-Yáñez, J. M. y Raj-Aryal, D. (2021). Efecto de la disposición de los residuos resultantes del beneficiado húmedo del café sobre las características físicas y química del agua de corriente natural. Terra Latinoamericana, 39, 1-11. e884. https://doi.org/10.28940/terra.v39i0.884 
discharged during the harvest. Sampling sites were San Pedro stream, Cabañas river, rancheria 30 de Agosto and Cuxtepec river. Water samples were obtained in October and December, 2016, and January, April and June, 2017. The following parameters were determined: $\mathrm{pH}$, electrical conductivity (EC), total dissolved solids (TDS), total suspended solids(TSS), sedimentable solids (SS), chemical oxygen demand (COD), biochemical oxygen demand (BOD), total nitrogen (TN), ammonia $\left(\mathrm{NH}_{4}\right)$, total phosphorus (TP), water hardness (WH), water temperature (WT), and air temperature (AT). COD and BOD content varied from site to site, with maximum values of 7.7 and $1.25 \mathrm{mg} \mathrm{L}^{-1}$, respectively. This low concentration allowed for high oxygen level (7.99 $\mathrm{mg} \mathrm{L}^{-1}$ ). The TDS load was $149.7 \mathrm{mg} \mathrm{L}^{-1}$ and $\mathrm{pH}$ was 8.9, indicating favorable conditions for aquatic life. In general, the values measured in groundwater under traditional coffee wet milling plants did not show levels that indicate risks for agricultural use or for consumption by animals and people inhabiting areas close to the water bodies in the region. These findings suggest a need to establish a contaminant monitoring system for the coffee-producing regions of Chiapas, and improve ecological management of residual waters produced by coffee wet milling according to pollutant concentrations.

Index words: water sources, mucilage, water properties, coffee pulp.

\section{INTRODUCCIÓN}

La planta de café constituye un elemento importante del paisaje, además genera condiciones ambientales, sociales y culturales especiales en el trópico de América Latina, este presenta altitudes propias para producir café con calidad en altitudes entre 1300 a 1800 m, estás regiones son ampliamente habitadas por millones de habitantes que se ganan la vida con este cultivo (CanetBrenes et al., 2016).

A nivel mundial se prevé que durante el ciclo 2020/21 la producción de café será de 9.1 millones de sacos (un saco equivale a $60 \mathrm{~kg}$ de café verde), para un total de 546000 toneladas, siendo inferior al año anterior, con 176.1 millones de sacos, equivalentes a 10566000 toneladas de café (USDA, 2020).

México en cambio, presenta sus mayores índices de producción de café en los estados de Chiapas (41.3\%), Veracruz (24.4\%), Puebla (15.8\%), Oaxaca
(8.2\%), Guerrero (4.5\%) e Hidalgo, San Luis Potosí, Nayarit, Jalisco, Colima, México, Tabasco, Querétaro y Morelos con menor índices de producción (5.9\%). Su mayor producción se obtiene con café arábica con $96 \%$ y robusta con $4 \%$ restante. Asimismo, se estima que en Chiapas durante el ciclo 2019/20 se obtuvo una producción de 252000 toneladas de café verde (CEDRSSA, 2019; SIAP, 2019), aunque de acuerdo con las estimaciones de SIAP (2020), se puede alcanzar una producción de 378601 toneladas en 253318 ha sembradas.

La región más sobresaliente en la producción de café en Chiapas, México incluye parte importante de la Sierra Madre, por lo tanto, incluye la Reserva de la Biosfera El Triunfo (ReBioTri). Está se caracteriza por poseer bosque de niebla, aportando servicios ecosistémicos de significativa relevancia, sobresaliendo la recarga y provisión de agua (López-Báez et al., 2014).

La producción de café incluye múltiples labores, tales como la siembra, renovación, cosecha y el beneficio húmedo, este último consiste en la separación de la pulpa o piel y mucilago del grano de café, usando un despulpador mecánico y altos volúmenes de agua (González, Vázquez y López, 2020). Esta práctica es frecuente en la zona cafetalera ReBioTri, debido a la disponibilidad de agua en la región. Pero los desechos resultantes, no son bien manejados y generan contaminación en las fuentes hídricas (MedinaMeléndez et al., 2016).

La pulpa y el mucilago son los principales subproductos del cerezo del café, compuestos de varias proteínas y azúcares en forma de pectinas, los que se fermentan por la acción de las enzimas que contienen las bacterias localizadas en el fruto del café. En este proceso, se liberan ácidos y compuestos químicos tóxicos como taninos, cafeína, fenoles, polifenoles y alcaloides que inhiben la actividad biológica de los microorganismos que degradan los subproductos del café (Ijanu, Kamaruddin y Norashiddin, 2019; Woldesenbet, Woldeyes y Chandravanshi, 2015). Durante la fermentación las levaduras y bacterias oxidan los azúcares parcialmente y generan energía Adenosín Trifosfato (ATP), etanol, ácido láctico, ácido acético, dióxido de carbono, propanol, butanol, ácido succínico, fórmico, butírico y substancias olorosas como aldehídos, cetonas, ésteres. Además, cambian el color, olor, densidad, acidez, pH, sólidos solubles, modifican la temperatura, composición química y microbiana del sustrato (Puerta, 2012). 
El agua resultante del beneficiado húmedo del café se convierte en un importante contaminante, ya que para el procesamiento de una tonelada de producto se necesitan $20000 \mathrm{~L}$ de agua (Rigueira, Lacerda, Matos, Donzeles y Palacin, 2010). Aunque la cantidad de agua varía según la región o el país, el consumo es alto. Sin embargo, el principal problema no radica en el elevado consumo de agua, sino en los impactos socioambientales asociados al vertido de sustancias en los cuerpos de agua, causando alteraciones del $(\mathrm{pH})$, sólidos suspendidos y demanda química de oxígeno (DQO); causando graves desequilibrios en la estructura de los ecosistemas, alterando los procesos biológicos del sistema hidrológico (Matuk-Velázco, Puerta y Rodríguez, 1997); además alteran el paisaje natural (Romero, Reyes, Dicovskiy y Pichardo, 2012'1), salud humana, flora, fauna acuática (Padmapriya, Tharian y Thirunalasundari, 2013) y efecto sobre la germinación de las semillas, desarrollo de raíces y alteración del ciclo celular de la lechuga (Lactuca sativa) por acción aneugénica (Louzada, Fonseca y de Oliveira, 2016).

Para tal caso, tanto el sistema orgánico como el convencional de producción de café, tienden a contaminar los cuerpos de agua. Por lo que, todos los subproductos derivados del beneficio húmedo del café provocan contaminación del agua superficial, afectando la biodiversidad y el paisaje (LópezCastillo, 2012). El objetivo de esta investigación fue analizar las propiedades físicas y químicas del agua de río Cuxtepec, río Cabañas y su principal afluente, donde son descargados de forma directa e indirecta los subproductos derivados del beneficiado húmedo del café durante el periodo de cosecha de la Sociedad de Producción Rural (SPR) "Unión Ramal Santa Cruz", Municipio de La Concordia, Chiapas, México.

\section{MATERIALES Y MÉTODOS}

\section{Sitios de Muestreo}

El área de estudio forma parte de la Reserva de la Biosfera El Triunfo, localizada entre las coordenadas $93^{\circ} 39^{\prime} 00^{\prime}$ y $92^{\circ} 52^{\prime} 00^{\prime \prime} \mathrm{O}$ y entre los $15^{\circ} 39^{\prime} 00^{\prime \prime}$ y $16^{\circ} 01^{\prime} 00^{\prime \prime} \mathrm{N}$, la altitud va desde los $600 \mathrm{~m}$ donde se localiza el embalse de la presa de almacenamiento
El Portillo II Juan Sabines Gutiérrez, a 2550 m en la cima del Cerro Quetzal (CEIGEG, 2020).

En esta zona geográfica se ubican las localidades estudiadas y fuentes de agua muestreadas, correspondientes al área representativa de la Sociedad de Producción Rural "Unión Ramal Santa Cruz" del municipio de La Concordia, Chiapas, México (Figura 1).

\section{Diseño Experimental}

La recolección y el beneficio húmedo de café en la región Sierra de Chiapas, comienza a finales de septiembre y continúa hasta marzo del año siguiente, derivado de lo anterior, se seleccionaron los sitios de muestreo arroyo San Pedro, río Cabañas, ranchería 30 de Agosto y río Cuxtepec para la caracterización física y química del agua.

El experimento se realizó mediante un diseño completamente aleatorizado compuesto por cuatro sitios (Cuadro 1) dentro de la misma subcuenca. Se muestreó de forma mensual en los meses y días de octubre $(13,14)$, diciembre $(14,15)$ del 2016 y enero $(12,13)$, abril $(15,16)$ y junio $(13,14)$ del 2017 , con un

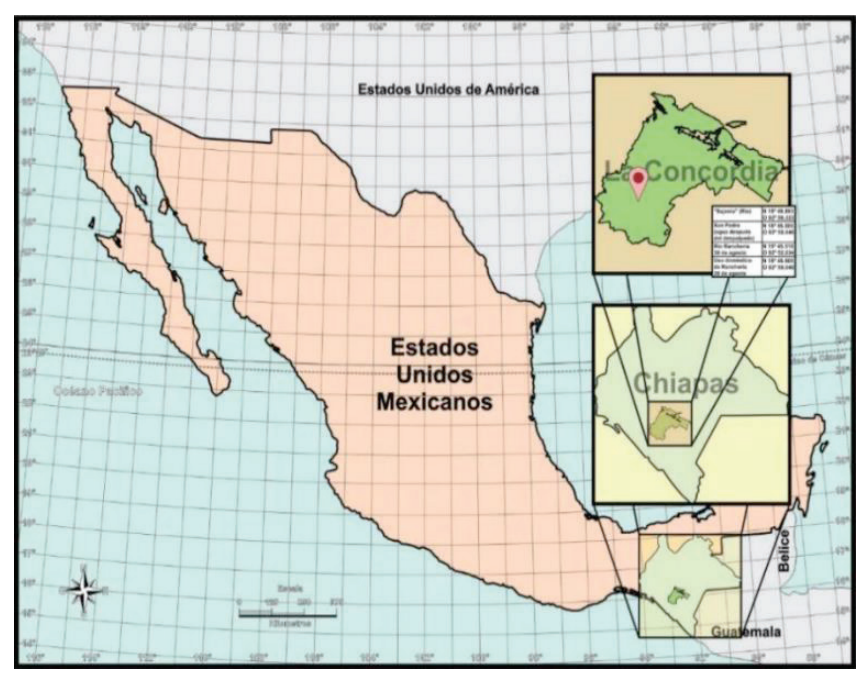

Figura 1. Ubicación de los sitios estudiados, señalando los puntos de muestreo. Fuente: elaborado por el autor.

Figure 1. Location of the sites studied indicating the sampling points. Source: prepared by the author.

\footnotetext{
${ }^{1}$ Romero Hernández, F. O., Reyes-Picado, O. D., Dicovskiy Rioboó, L. M., \& Pichardo Hernández, C. B. (2012). Caracterización de tres beneficios húmedos colectivos y uno industrializado de café con énfasis en una propuesta de mejora de un modelo de beneficio húmedo colectivo en la Unión de Cooperativa Agropecuaria del Norte UCANOR, Jinotega. Informe de Final. Universidad Nacional de Ingeniería de Nicaragua. Consultado el 22 de febrero, 2021 , desde http://ribuni.uni.edu.ni/1281/1/39805.pdf
} 
total de 20 muestras colectadas y distribuidas durante la época de cosecha del café. La distancia entre sitios de muestreo fue de aproximadamente un $\mathrm{km}$ a la redonda, esto aseguró que las condiciones ambientales fueran relativamente homogéneas, así como las prácticas de beneficiado húmedo.

\section{Muestras de Agua}

En los cuatro sitios de muestreo (Cuadro 1) la toma de muestras de agua fue similar en todo el proceso, se obtuvieron a un intervalo de tiempo, que fue de las 11:00 - 13:00 AM (Álvarez, Smeltekop, Cuba y Loza, 2011). Para cada sitio se colectaron en forma manual dos litros de agua en botellas de plástico y cristal esterilizadas, que fueron introducidas bajo la superficie, a la profundidad de $25 \mathrm{~cm}$ (INVEMAR, 2003), sumergidas en dirección opuesta a la corriente de agua y tapadas inmediatamente después de haberse llenado e identificadas. Se mantuvieron a $4{ }^{\circ} \mathrm{C}$ en hielo durante su transportación para disminuir la degradación de los componentes de la muestra, hasta su análisis en el laboratorio, tal como lo establece American Public Health Association (Eaton, Clesceri, Rice y Greenberg, 2005) sobre estándares de calidad de aguas residuales, consumo y norma sobre calidad de agua (NMX-AA051-SCFI-2016, 2016).

\section{Análisis Químico de las Muestras de Agua}

Las mediciones realizadas in situ fueron $(\mathrm{pH})$, temperatura del agua (TA), conductividad eléctrica (CE) y sólidos totales disueltos (STD) con un equipo digital multímetro portátil marca Hatch Modelo Sensión ${ }^{\mathrm{TM}} 156$.
Los análisis se realizaron en el laboratorio del Colegio de la Frontera Sur, ubicado en la ciudad de San Cristóbal de las Casas, Chiapas. Se analizó demanda química de oxígeno (DQO) por el método espectrofotométrico/ reflujo cerrado Hach (método 10212), amonio $\left(\mathrm{NH}_{4}^{+}\right)$, amoniaco $\left(\mathrm{NH}_{3}\right)$, nitrógeno total $(\mathrm{NT})$, se midieron por colorimetría utilizando un espectrofotómetro (DR/2010 $\mathrm{HACH}$, Loveland, EE.UU.) según las instrucciones de $\left(\mathrm{HACH}, 1999^{2}\right)$, demanda bioquímica de oxígeno (DBO), se midió por métodos estándar (APHA, 1998), sólidos suspendidos totales (SST), se determinó de acuerdo con (Luthy, 1978), fósforo total (PT) con la Norma Mexicana NMX-AA-029-SCFI-2001 (2001), dureza del agua (DA) y sólidos sedimentables (SS), por el método recomendado por Baird, Eaton y Rice (2017). Por cada determinación en el estudio se hizo un análisis por triplicado en el laboratorio.

\section{Análisis Estadístico}

Los datos originales fueron analizados obteniéndose un coeficiente de variación mayor de $30 \%$ en 10 parámetros, por lo que se decidió hacer una transformación logarítmica $\log (\mathrm{X}+1)$ cuando existieron valores menores de 1 y raíz cuadrada $X=\sqrt{x+0.5}$ con valores de cero, con ello se ajustó un coeficiente de variación menor para los distintos parámetros de estudio.

Los datos de los parámetros estudiados durante la investigación fueron analizados, utilizando el programa estadístico Statgraphics Centurion XVIII 2013. El análisis de varianza y pruebas de medias de los parámetros que presentaron distribución normal se realizaron siguiendo un análisis de varianza de una vía

Cuadro 1. Tipos de agua muestreada y ubicación geográfica del área de toma de muestras.

Table 1. Types of water sampled and geographic location of the sampling area.

\begin{tabular}{|c|c|c|c|c|}
\hline Sitio de muestreo & Tipo de fuente de agua muestreada & Altitud (m) & Latitud Norte & Longitud Oeste \\
\hline San Pedro & Arroyo, agua de corriente & 1000 & $15^{\circ} 45^{\prime} 50^{\prime \prime}$ & $92^{\circ} 58^{\prime} 04^{\prime \prime}$ \\
\hline Finca 30 de Agosto & Río Cabañas, agua de corriente & 910 & $15^{\circ} 45^{\prime} 30^{\prime \prime}$ & $92^{\circ} 58^{\prime} 00^{\prime \prime}$ \\
\hline Ranchería 30 de Agosto & Agua de uso doméstico, agua entubada & 1050 & $15^{\circ} 45^{\prime} 11^{\prime \prime}$ & $92^{\circ} 58^{\prime} 11^{\prime \prime}$ \\
\hline Rancho Sajonia & Río Cuxtepec, agua de corriente & 700 & $15^{\circ} 49^{\prime} 53^{\prime \prime}$ & $92^{\circ} 56^{\prime} 20^{\prime \prime}$ \\
\hline
\end{tabular}

Fuente: Elaborado por el autor.

Source: Prepared by the author.

2 HACH-DR/2010 spectrophotometer procedures manual https://www.fieldenvironmental.com/assets/files/Literature/HACH\%20Spectrophotometer\%20 DR2010\%20Procedures\%20Guide.pdf 
y cuando se encontraron diferencias significativas $(P \leq 0.05)$ se aplicó la prueba múltiple de medias Tukey $(P \leq 0.05)$; y cuando los datos no provenían de una distribución normal (prueba de Shapiro-Wilks), se aplicó el análisis de varianza no paramétrico de Kruskal-Wallis (Kruskal y Wallis, 1952), que evalúa la hipótesis nula de igualdad para las medianas de los diferentes sitios de muestreo para cada variable de respuesta.

Asimismo, los promedios de los valores de los parámetros estudiados fueron comparado con las normas de referencia que establecen los criterios ecológicos, límites máximos permisibles para el consumo humano, animales domésticos y uso agrícola, estas fueron: US Environmental Protection Agency (US-EPA, 1986) sobre criterios sobre calidad de agua; Secretaria de Desarrollo Urbano y Ecología (SEDUE, 1989) sobre criterios ecológicos; Norma Oficial Mexicana (NOM-127-SSA1-1994, 1994) sobre usos del agua; Norma Oficial Mexicana (NOM001-ECOL-1996) sobre contaminantes en aguas de descarga; Norma Oficial Mexicana (NOM-011CONAGUA-2015, 2015) sobre conservación del recurso agua; Norma Mexicana (NMX-AA-051-SCFI-2016, 2016) sobre análisis de agua y Secretaría de Medio Ambiente y Recursos Naturales - Comisión Nacional del Agua SEMARNAT-CONAGUA (2019) sobre Ley Federal de Derechos Disposiciones Aplicables en materia de aguas nacionales.

\section{RESULTADOS Y DISCUSIÓN}

\section{Parámetros Fisicoquímicos del Agua de la Zona Cafetalera}

Los resultados promedios obtenidos de los parámetros $\mathrm{DBO}, \mathrm{NH}_{3}, \mathrm{SDT}, \mathrm{SST}, \mathrm{NO}_{3}^{-}, \mathrm{NO}_{2}^{-}, \mathrm{PT}, \mathrm{NT}$, $\mathrm{NH}_{3}, \mathrm{SS}, \mathrm{CaCO}_{3}$, temperatura del agua, temperatura ambiental, $\mathrm{pH}, \mathrm{CE}, \mathrm{OD}$; revelaron valores por debajo de los niveles máximos permisibles por la Norma Oficial Mexicana NOM-127-SSA-1994 (1994), Norma Oficial Mexicana NOM-001-ECOL-1996, (1996) y US Environmental Protection Agency (US-EPA, 1986), para ser utilizados en agua para riego agrícola, uso público o urbano; y para la cría de especies acuáticas resultaron inferiores (Cuadro 2 y 3 ).

El parámetro DQO no mostró diferencia estadística significativa entre sitios muestreados. Sin embargo, los niveles fueron superiores a los permisibles de $3.0 \mathrm{mg} \mathrm{L}^{-1}$ US Environmental Protection Agency (US EPA, 1986) y 1-2 mg L-1 Norma Oficial Mexicana (NOM-001-ECOL-1996, 1996) con valor promedio entre los sitios de $6.4 \mathrm{mg} \mathrm{L}^{-1}$ (Cuadro 2), aunque inferiores a los 831 a $3244 \mathrm{mg} \mathrm{L}^{-1}$ y $1282967 \mathrm{mg} \mathrm{L}^{-1}$ de DQO y DBO, respectivamente en agua de corriente (Tekle, Hailu, Wassie y Tesema, 2015) en seis sitios de Etiopía, así como los $5683 \mathrm{mg} \mathrm{L}^{-1}$ reportados por Tsigereda, Metadel, Mekibib y Fisseha (2013) y $1500 \mathrm{mg} \mathrm{L}^{-1}$ en Quindío Colombia (Torres-Valenzuela, Sanín, Arango y Serna, 2019). Asimismo, las concentraciones encontradas 5, 7.7, 7.7 y $5.2 \mathrm{mg} \mathrm{L}^{-1}$ en promedio, fueron superiores a las reportadas por Chave (2007), quien establece que la cantidad debe ser menor a $3 \mathrm{mg} \mathrm{L}^{-1}$ para favorecer la vida acuática.

Respecto a la DBO, con valor promedio entre las medias de los sitios de $0.9 \mathrm{mg} \mathrm{L}^{-1}$ comparada con la DQO de 5, 7.7, 7.7 y $5.2 \mathrm{mg} \mathrm{L}^{-1}$, se obtuvo una relación de DQO:DBO superior a 5:1, esto indica una baja biodegradación de los residuos orgánicos en el agua, ya que de acuerdo con Woldesenbet, Woldeyes, Singh y Chandravanshi (2014), dicha relación debe ser inferior a 5:1 para observar alto grado de biodegradación de los residuos. Ahora bien, la DBO obtenida, resulta importante ya que, es menor a lo establecido por la Secretaría de Medio Ambiente y Recursos Naturales - Comisión Nacional del Agua (SEMARNATCONAGUA, 1996), donde los límites máximos permisibles son 200,150 y $60 \mathrm{mg} \mathrm{L}^{-1}$, para uso en riego agrícola, uso público urbano y protección de vidas acuáticas, respectivamente.

El valor promedio de DQO obtenido, pudo deberse a los desechos derivados del beneficio húmedo de café, aunque pudiera haber una aportación de remanentes orgánicos de especies vegetales nativas del área de estudio, como algas y hiervas en las aguas superficiales donde se observó una alta diversidad de estas especies, ya que de acuerdo con Minuta y Jini (2017), la presencia de sustancias orgánicas en el agua en zonas cafetaleras puede crear niveles considerables de DBO. Pero también la variación en las concentraciones de (DQO), según Dadi et al. (2018), esto podría ocurrir debido a las diferencias en la capacidad de despulpado de los molinos, el tiempo de fermentación, la cantidad de agua utilizada durante de lavado y la fermentación del grano del café. 
Cuadro 2. Valores medios de las propiedades fisicoquímicas de las muestras de agua de diferentes sitios de muestreo en la zona cafetalera de la SPR "Unión Ramal Santa Cruz" del municipio de La Concordia, Chiapas, México.

Table 2. Physical-chemical properties of water samples from different sampling sites in the coffee-growing zone of SPR "Unión Ramal Santa Cruz" in La Concordia municipality, Chiapas, Mexico.

\begin{tabular}{lrrrr}
\hline & \multicolumn{3}{c}{ Sitio de muestreo } \\
\cline { 2 - 5 } Parámetros fisicoquímicos & Arroyo San Pedro & Río Cabañas & $\begin{array}{c}\text { Uso doméstico de } \\
\text { ranchería 30 de Agosto }\end{array}$ & Río Cuxtepec \\
\hline DQO $\left(\mathrm{mg} \mathrm{L}^{-1}\right)$ & 5 & 7.7 & 7.7 & 5.2 \\
$\mathrm{DBO}\left(\mathrm{mg} \mathrm{L}^{-1}\right)$ & 0.6 & 1.25 & 0.7 & 1.05 \\
$\mathrm{NH}_{3}\left(\mathrm{mg} \mathrm{L}^{-1}\right)$ & 0 & 0.1 & 0.008 & 0.03 \\
$\mathrm{NH}_{4}^{+}\left(\mathrm{mg} \mathrm{L}^{-1}\right)$ & 0.02 & 0.06 & 0.02 & 0.04 \\
$\mathrm{SDT}_{\left(\mathrm{mg} \mathrm{L}^{-1}\right)}$ & 147.54 & 76.84 & 149.7 & 67.22 \\
$\mathrm{SST}\left(\mathrm{mg} \mathrm{L}^{-1}\right)$ & 4.8 & 4.07 & 3.33 & 8.6 \\
$\mathrm{PT}\left(\mathrm{mg} \mathrm{L}^{-1}\right)$ & 0.66 & 0.446 & 0.0 & 0.46 \\
$\mathrm{NT}\left(\mathrm{mg} \mathrm{L}^{-1}\right)$ & 1.7 & 2.35 & 0.0 & 1.04 \\
$\mathrm{SS}\left(\mathrm{mL} \mathrm{L}^{-1}\right)$ & 0.2 & 0.1 & 0.07 & 0.1 \\
$\mathrm{CaCO}$ & $\left(\mathrm{mg} \mathrm{L}^{-1}\right)$ & 90.93 & 173 & 82.8 \\
$\mathrm{~T}$. agua $\left({ }^{\circ} \mathrm{C}\right)$ & 141.8 & 23.2 & 26.14 & 26.54 \\
$\mathrm{~T}$. ambiente $\left({ }^{\circ} \mathrm{C}\right)$ & 22.66 & 26.2 & 26.8 & 27.8 \\
$\mathrm{pH}$ & 25 & 8.52 & 8.19 & 8.88 \\
$\mathrm{CE} \mu \mathrm{cm}$ & 8.45 & 156.24 & 319.4 & 147.14 \\
$\mathrm{OD}\left(\mathrm{mg} \mathrm{L}^{-1}\right)$ & 293.8 & 5.16 & 5.66 & 6.25 \\
\hline
\end{tabular}

$\mathrm{DQO}=$ demanda química de oxígeno; $\mathrm{DBO}=$ demanda bioquímica de oxígeno; $\mathrm{NH}_{3}=$ amoniaco $; \mathrm{NH}_{4}{ }^{+}=$amonio; $\mathrm{SDT}=$ sólidos disueltos totales; $\mathrm{SST}=$ sólidos suspendidos totales; $\mathrm{PT}=$ fósforo total; $\mathrm{NT}=$ nitrógeno total; $\mathrm{SS}=$ sólidos sedimentables; $\mathrm{CaCO}_{3}=$ carbonato de calcio (dureza del agua); $\mathrm{CE}=$ conductividad eléctrica; $\mathrm{OD}=$ oxígeno disuelto.

$\mathrm{DQO}=$ chemical oxygen demand; $\mathrm{DBO}=$ biochemical oxygen demand; $\mathrm{NH}_{3}=$ ammonia; $\mathrm{NH}_{4}^{+}=$ammonium; $\mathrm{SDT}=$ total dissolved solids; $\mathrm{SST}=$ total suspended solids; $\mathrm{PT}=$ total phosphorus; $\mathrm{NT}=$ total nitrogen; $\mathrm{SS}=$ settling solids; $\mathrm{CaCO}_{3}=$ calcium carbonate (water hardness); $\mathrm{CE}=$ electrical conductivity; $\mathrm{OD}=$ dissolved oxygen

Las concentraciones SST de 4.8,4.07, 3.33, $8.6 \mathrm{mg} \mathrm{L}{ }^{-1} ; \mathrm{CaCO}_{3} 141.8,90.93,173,82.8 \mathrm{mg} \mathrm{L}^{-1}$; temperatura del agua $22.66,23.2,26.14,26.14^{\circ} \mathrm{C}$, y OD 4.82,5.16, 5.66, $6.25 \mathrm{mg} \mathrm{L}^{-1}$ para los sitios de muestreo arroyo San Pedro, río Cabañas, agua de uso doméstico y río Cuxtepec respectivamente, no mostraron diferencia estadística $(P>0.05$, Cuadro 3). Aunque al comparar con los niveles permisibles en agua para riego agrícola, uso público o urbano, y cría de especies acuáticas, estos parámetros resultaron menores a los niveles recomendados por las normas de referencia: US Environmental Protection Agency (US-EPA, 1986); Norma Oficial Mexicana NOM-127-SSA-1-1994 (1994); Norma Oficial Mexicana NOM-001ECOL-1996, (1996); Norma Oficial Mexicana NOM011-CONAGUA-2015 (2015) y Secretaría de Medio Ambiente y Recursos Naturales-Comisión Nacional del Agua (SEMARNAT-CONAGUA, 2019).

\section{Sólidos Disueltos Totales (SDT), Fósforo Total (PT), pH y Conductividad Eléctrica (CE) en Agua}

Para el caso de SDT, se observó diferencia estadística significativa $(P \leq 0.05)$, siendo los sitios de muestreo río Cuxtepec y arroyo San Pedro, los que resultaron con mayores concentraciones, seguidos por el río Cabañas y ranchería 30 de Agosto (Cuadro 3). Las cantidades de SDT en muestras provenientes de San Pedro y ranchería 30 de Agosto, fue en promedio 147.54 y $149.7 \mathrm{mg} \mathrm{L}^{-1}$, respectivamente. Estas concentraciones están sobre el promedio detectado en los ríos de todo el mundo de $120 \mathrm{mg} \mathrm{L}^{-1}$ (Livingston, 1963). Las concentraciones obtenidas evidencian que los puntos de muestreo definidos para la investigación, cumplen con lo establecido en la Norma Oficial Mexicana NOM-127SSA1-1994 (1994), está considera $1000 \mathrm{mg} \mathrm{L}^{-1}$ como límites permisibles para consumo humano del agua. 
Cuadro 3. Concentraciones promedios de los parámetros físicos y químicos en los sitios de muestreo, evaluados durante el periodo del beneficiado húmedo de café en la Sociedad de Producción Rural la Ramal, municipio de La Concordia, Chiapas, México.

Table 3. Average concentrations of physical and chemical parameters in the sampling sites evaluated during coffee wet milling in the Ramal Rural Production Society, La Concordia municipality, Chiapas, Mexico.

\begin{tabular}{lccccccccc}
\hline \multirow{2}{*}{ Sitio de muestreo } & \multicolumn{8}{c}{ Parámetros medidos } \\
\cline { 2 - 10 } & DQO & SDT & SST & PT & $\mathrm{CaCO}_{3}$ & T. agua & $\mathrm{pH}$ & $\mathrm{CE}$ & $\mathrm{OD}$ \\
\hline & & & $\mathrm{mg} \mathrm{L}^{-1}$ & & & ${ }^{\circ} \mathrm{C}$ & & $\mu \mathrm{S} / \mathrm{cm}^{\prime}$ & $\mathrm{mg} \mathrm{L}^{-1}$ \\
Arroyo San Pedro & $5.6 \mathrm{ab}^{\dagger}$ & $147.7 \mathrm{a}$ & $4.8 \mathrm{a}$ & $0.66 \mathrm{a}$ & $141.8 \mathrm{a}$ & $22.7 \mathrm{a}$ & $8.45 \mathrm{bc}$ & $293.8 \mathrm{a}$ & $4.82 \mathrm{a}$ \\
Río Cabañas & $1.47 \mathrm{~b}$ & $83.64 \mathrm{~b}$ & $4.06 \mathrm{a}$ & $0.44 \mathrm{a}$ & $91 \mathrm{~b}$ & $23.1 \mathrm{a}$ & $8.51 \mathrm{~b}$ & $156.3 \mathrm{~b}$ & $5.16 \mathrm{a}$ \\
Uso doméstico & $8.6 \mathrm{a}$ & $67.24 \mathrm{~b}$ & $3.33 \mathrm{a}$ & $0.01 \mathrm{~b}$ & $172.2 \mathrm{a}$ & $26.1 \mathrm{a}$ & $8.19 \mathrm{c}$ & $319.4 \mathrm{a}$ & $5.66 \mathrm{a}$ \\
Río Cuxtepec & $5.2 \mathrm{ab}$ & $149.7 \mathrm{a}$ & $8.6 \mathrm{a}$ & $0.53 \mathrm{a}$ & $83 \mathrm{~b}$ & $26.5 \mathrm{a}$ & $8.9 \mathrm{a}$ & $147.2 \mathrm{~b}$ & $6.25 \mathrm{a}$ \\
CV (\%) & 50.5 & 35.9 & 42.1 & 16.9 & 9.3 & 15.7 & 3.6 & 8.0 & 24.1 \\
EE & 0.41 & 6.43 & 0.15 & 0.03 & 0.04 & 1.67 & 0.09 & 19.28 & 0.58 \\
\hline
\end{tabular}

$\mathrm{DQO}=$ demanda química de oxígeno; $\mathrm{SDT}=$ sólidos disueltos totales; $\mathrm{SST}=$ sólidos suspendidos totales; $\mathrm{PT}=$ fósforo total; $\mathrm{CaCO}_{3}=$ carbonato de calcio (dureza del agua); $\mathrm{T}$. agua = temperatura del agua; $\mathrm{CE}=$ conductividad eléctrica; $\mathrm{OD}=$ oxígeno disuelto; $\mathrm{CV}=$ coeficiente de variación; $\mathrm{EE}=$ error estándar. ${ }^{\dagger}$ Medias con letras distintas son significativamente diferentes, según la prueba de Tukey (HSD) $(P \leq 0.05)$.

$\mathrm{DQO}=$ chemical oxygen demand; $\mathrm{SDT}=$ total dissolved solids; $\mathrm{SST}=$ total suspended solids; $\mathrm{PT}=$ total phosphorus; $\mathrm{CaCO}_{3}=\mathrm{calcium}$ carbonate $($ water hardness); $\mathrm{T}$. water $=$ water temperature; $\mathrm{CE}=$ electrical conductivity; $\mathrm{OD}=$ dissolved oxygen; $\mathrm{CV}=$ coefficient of variation; $\mathrm{EE}=$ standard error. ${ }^{\dagger} \mathrm{Means}$ with different letters are significantly different according to Tukey's test (HSD) $(P \leq 0.05)$.

La concentración de PT fue estadísticamente diferente entre sitios de muestreo $(P \leq 0.05$, Cuadro 3), observándose para el agua de ranchería 30 de Agosto, durante todo el periodo de investigación $0.01 \mathrm{mg} \mathrm{L}^{-1}$ de residuos de este elemento, frente a los tres sitios restantes que fluctuó entre 0.44 a $0.66 \mathrm{mg} \mathrm{L}^{-1}$.

La concentración PT obtenidas en tres de los cuatro sitios de muestreo estuvo dentro del intervalo de los límites permisibles por debajo de $5 \mathrm{mg} \mathrm{L}^{-1}$ (Minuta y Jini, 2017; CE-CCA-001/89, 1989³; Norma Mexicana NMX-AA-051-SCFI-2016, 2016; SEMARNATCONAGUA, 2019) y también del límite máximo superior para riego agrícola $30 \mathrm{mg} \mathrm{L}^{-1}$, uso urbano $30 \mathrm{mg} \mathrm{L}^{-1}$ y para la protección de vida acuática $10 \mathrm{mg} \mathrm{L}^{-1}$ mensual, respectivamente de acuerdo con la Norma Oficial Mexicana NOM-001-ECOL-1996 (1996) y US-EPA (1986).

Las concentraciones de PT entre sitios, presentaron diferencia estadística significativa $(P \leq 0.05$, Cuadro 3$)$, sin embargo, no resultan tóxicas para la salud acuática y humana. Las cantidades encontradas aquí podrían deberse a que en el efluente las cantidades de PT fueron bajas para producir un cambio en las concentraciones de los sitios de muestreo y otra posibilidad es que el PT se pudo haber hidrolizado en otras formas. Además de que el $\mathrm{P}$ es un compuesto altamente dinámico en el agua y puede sufrir una transformación química y ser almacenado por diversos organismos acuáticos (US-EPA, 2008).

El pH presentó diferencias estadísticas entre sitios muestreados (Cuadro 3). Los niveles de $\mathrm{pH}$ fueron mayores en río Cuxtepec (8.9), seguido por río Cabañas (8.51) y San Pedro (8.45) y el valor más bajo se observó en ranchería 30 de Agosto (8.19). Estos resultados son diferentes a los obtenidos por Tekle et al. (2015) con $\mathrm{pH}$ promedios de 7.1 en agua de corriente en Etiopía que recibían cargas provenientes de plantas de beneficio húmedo de café. En esta región a pesar de que la mayoría de los productores de café arrojan los desechos del beneficiado húmedo cerca de las orillas de las fuentes hídricas, los niveles de $\mathrm{pH}$ resultaron ligeramente alcalinos, encontrándose al límite de los criterios ecológicos para consumo humano, en un rango de 6.5 a 8.5 , mientras que para uso agrícola resultó favorable de acuerdo con los niveles recomendados de 6.0 a 9.0 (US-EPA, 1986); Criterios Ecológicos de

\footnotetext{
${ }^{3}$ Acuerdo por el que se establecen los criterios ecológicos de calidad del agua CE-CCA-001/89. DOF. Consultado el 2 de junio 2020, desde http://legismex.mty. itesm.mx/acu/acca001.pdf
} 
Calidad del Aguas (CE-CCA-001/89, 1989³); Norma Oficial Mexicana (NOM-127-SSA1-1994, 1994); Norma Oficial Mexicana (NOM-001-ECOL-1996, 1996). Por consiguiente, los valores de $\mathrm{pH}$, pueden clasificarse como medianamente alcalino, con calidad para consumo humano y riego agrícola. Esta variable es importante para indicar la calidad del agua potable (Rajendran y Mansiya, 2015) y varía con el contenido de gases y sólidos en el agua (Patil, Sawant y Deshmukh, 2012). La concentración del $\mathrm{pH}$ observada sugiere bajas concentraciones de desechos orgánicos provenientes del beneficio húmedo del café, que pudieran causar la oxidación suficiente para disminuir el $\mathrm{pH}$ por debajo de 6.5-8.5 (WHO, 1995).

La conductividad eléctrica (CE) del agua de ranchería 30 de Agosto fue de $319.4 \mu \mathrm{S} \mathrm{cm}^{-1}$ y en San Pedro de $293.8 \mu \mathrm{S} \mathrm{cm}^{-1}$, siendo estadísticamente superiores al resto de sitios muestreados $(P<0.05$, Cuadro 3). Este parámetro es el más importante para evaluar la calidad del agua de riego, es la concentración de sales que comúnmente se mide como $\mathrm{CE}$; si es mayor a $3000 \mu \mathrm{S} \mathrm{cm} \mathrm{cm}^{-1}$ puede afectar al crecimiento de varios cultivos (Ayers y Westcot, 1987). En esta investigación la mayor CE se encontró en muestras de ranchería 30 Agosto con $319.4 \mu \mathrm{S} \mathrm{cm}^{-1}$ y arroyo San Pedro con $293.8 \mu \mathrm{S} \mathrm{cm}^{-1}$, estos valores son similares a los reportados por Dadi et al. (2018).

El oxígeno disuelto (OD) en el agua resulto estadísticamente similar en los cuatro sitios de muestreo $(P<0.05$, Cuadro 3$)$, encontrándose estas concentraciones dentro del intervalo considerado como valores máximos permisibles por las normas de referencia (US-EPA, 1986) de 4-9.5 y por la Norma Oficial Mexicana NOM-001-ECOL-1996 (1996) con 2-7.8 $\mathrm{mg} \mathrm{L}^{-1}$.

Las concentraciones encontradas de OD en el agua, en los cuatro sitios de muestreo, presupone que se debe a la aportación de productos orgánicos por la vegetación y algunos otros organismos presentes en la zona. En este sentido Rodier, Legube y Merlet (2011), sostienen que el principal factor que contribuye a los cambios en los niveles de OD, es el incremento de residuos orgánicos en la fuente acuática, mientras que el decaimiento de éstos, conlleva al consumo de oxígeno y ocurre frecuentemente en el verano, cuando los animales acuáticos requieren de más oxígeno para soportar altos niveles de metabolismo.

En el análisis de varianza mediante la prueba de Kruskal-Wallis de los parámetros $\mathrm{NT}, \mathrm{DBO}, \mathrm{SS}, \mathrm{NH}_{4}^{+}$,
$\mathrm{NH}_{3}$ y temperatura ambiente, se obtuvieron valores de probabilidad mayores al 5\% $(P>0.05)$, para los sitios de muestreo arroyo San Pedro, río Cabañas, agua de uso doméstico y río Cuxtepec. Por lo tanto, no se encontraron diferencias significativas entre sitios muestreados para estas variables de respuesta. Asimismo, las concentraciones de estos parámetros están por debajo de los límites superiores considerados por las normas de referencia de US Environmental Protection Agency (US-EPA, 1986) y Norma Oficial Mexicana (NOM-001-ECOL-1996, 1996).

Las concentraciones de $\mathrm{NH}_{4}^{+}$encontradas en los cuatro sitios de muestreo (Cuadro 2), arroyo San Pedro, ranchería 30 de Agosto, río Cabañas y río Cuxtepec podrían ser tóxicas para algunas especies acuáticas de acuerdo a los límites permisibles para la mayoría de especies de animales acuáticos que va de 0. 0017 a $22.8 \mathrm{mg} \mathrm{L}^{-1}$, sobre todo para salmónidos (US-EPA, 1986).

La cantidad de $\mathrm{NH}_{3}$ no ionizado, corresponde a una importante fuente de energía, pero también una forma tóxica relevante, la concentración de 0.083 a $4.60 \mathrm{mg} \mathrm{L}^{-1}$ ha afectado severamente a 29 especies de nueve familias y 18 géneros de peces. Además, existen reportes en otras nueve especies de cinco familias y siete géneros susceptibles a dosis que van de 0.0017 a $0.612 \mathrm{mg} \mathrm{L}^{-1}$ de $\mathrm{NH}_{3}$, siendo los más afectados el grupo de los salmónidos (US-EPA, 1986). Por lo que corresponde al $\mathrm{NH}_{3}$ encontrado en promedios de $0.1 \mathrm{mg} \mathrm{L}^{-1}$ en río Cabañas, $0.008 \mathrm{mg} \mathrm{L}^{-1}$ en ranchería 30 de Agosto y $0.03 \mathrm{mg} \mathrm{L}^{-1}$ en río Cuxtepec, están en los límites permisibles para el uso de consumo animal, pero no para especies acuáticas como las señaladas anteriormente, aunque favorable para consumo humano por la ausencia de este compuesto en el agua del arroyo San Pedro (Cuadro 2).

Aunque la mayoría de los productores realizan el beneficio húmedo del café a una distancia que va entre los 200 a $400 \mathrm{~m}$ de las fuentes de agua muestreadas, otros lo realizan en sus hogares con agua entubada potable y dispersados al suelo, llegando éstos a las corrientes de los ríos, diluidos y degradados al inicio del periodo de lluvias. Generalmente dos meses después de la actividad de despulpado del café, por lo cual es probable que esa sea una de las razones de las bajas concentraciones encontradas de los contaminantes, lo que resulta inofensivo para el consumo humano y uso agrícola, según Secretaría de Medio Ambiente y Recursos Naturales - Comisión Nacional del 
Agua (SEMARNAT-CONAGUA, 1996), ya que las cantidades encontradas no superaron los niveles mínimos permitidos por las diferentes normas citadas (Cuadro 2).

\section{CONCLUSIONES}

Los componentes residuales producto del beneficio húmedo del café determinados en los cuerpos de agua de la Sociedad de Producción Rural La Ramal en el municipio de La Concordia, Chiapas, resultaron ser menores a los límites considerados como dañinos a la salud humana, animal y para riego agrícola.

La proporción de DQO (demanda química de oxígeno) y DBO (demanda bioquímica de oxígeno) fueron superiores a 5:1, lo que indica que hay una baja biodegradación de los residuos orgánicos en el agua. Así como un $\mathrm{pH}$ relativamente alcalino que va desde 8.2 a 8.9. Los contenidos de PT (fósforo total) se consideran altos, lo que podría constituirse en un riesgo de eutrofización, en tanto que la CE (conductividad eléctrica), uno de los factores que determina la calidad del agua de riego para la agricultura, se obtuvieron niveles bajos, siendo aptos para cualquier cultivo agrícola.

A pesar de las bajas concentraciones de los residuos obtenidos hasta ahora, es importante considerar el eventual riesgo de los contaminantes procedentes del despulpado del café, ya que al acumularse resultan dañinos a la salud humana. También afectan las funciones ecológicas del ecosistema, ponen en riesgo las contribuciones económicas estatales y nacionales. Por esto, se requiere realizar monitoreos frecuentes durante los subsiguientes ciclos de cultivo del café.

\section{DECLARACIÓN DE ÉTICA}

No aplica.

\section{CONSENTIMIENTO PARA PUBLICACIÓN}

No aplica.

\section{DISPONIBILIDAD DE DATOS}

Los conjuntos de datos utilizados o analizados durante el estudio actual están en disponibilidad del autor a solicitud razonable.

\section{CONFLICTO DE INTERESES}

Los autores declaran que no tienen intereses en competencia.

\section{FONDOS}

Este estudio se realizó con el apoyo de la SES DGESUI - DFI - PRODEP, para el fortalecimiento de Cuerpos Académicos.

\section{CONTRIBUCIÓN DE LOS AUTORES}

Preparación del borrador original y levantamiento de información: R.E.R.N. Revisión del borrador original y metodología: J.A.M.M. Revisión del borrador original análisis químico de las muestras: J.C. de la T. Revisión del borrador original y de los cálculos realizados: G.R.E. Levantamiento de información revisión de borrador original campo y revisión de bases de datos: J.M.S.Y. Planeación y revisión del borrador original: D.R.A.

\section{AGRADECIMIENTOS}

A la SES - DGESUI - DFI - PRODEP, por el financiamiento del proyecto para el fortalecimiento de Cuerpos Académicos.

\section{LITERATURA CITADA}

Álvarez, J., Smeltekop, H., Cuba, N., \& Loza-Murguia, M. (2011). Evaluación de un sistema de tratamiento de aguas residuales del prebeneficiado de café (Coffea arabica) implementado en la comunidad Carmen Pampa provincia Nor Yungas del Departamento de La Paz. Journal of the Selva Andina Research Society, 2(1), 34-42.

Ayers, R. S., \& Westcot, D. W. (1987). La calidad del agua y su uso en la agricultura. Estudio FAO. Riego y Drenaje no. 29. Roma: FAO.

Baird, R. B., Eaton, A. D., \& Rice, E. W. (2017). Standard method for examination of water and wastewater $\left(23^{\text {th }} \mathrm{ed}.\right)$. Consultado el 24 de octubre, 2019, desde https://www. academia.edu/38769108/

Canet-Brenes, G., Soto-Víquez, C., Ocampo-Thompson, P., Rivera-Ramírez, J., Navarro-Hurtado, A., GuatemalaMorales, G. M., \& Villanueva-Rodríguez, S. (2016). La situación y tendencias de la producción de café en América Latina y El Caribe. San José, C.R.: IICA-CIATEJ. ISBN: 97892-9248-651-8. 
CEDRSSA (Centro de Estudios para el Desarrollo Rural Sustentable y la Soberanía Alimentaria). (2019). Investigación interna. Comercio Internacional del café, el caso de México. Consultado el 24 de octubre, 2020, desde http://www.cedrssa. gob.mx/post_n-comercio_internacional_del_cafn-n-_el_ caso_de_mn-xico.htm

CEIGEG $^{-}$(Comité Estatal de Información Estadística y Geográfica). (2020). Geoweb Chiapas 3.0. Consultado el 2 de junio 2020, desde http://map.ceieg.chiapas.gob.mx/geoweb/

Chave, P. (2007). The EU water framework directive. Volume 6. UK: IWA Publishing. https://doi.org/10.2166/9781780402239

Dadi, D., Mengistie, E., Terefe, G., Getahun, T., Haddis, A., Birke, W.,... Van der Bruggen, B. (2018). Assessment of the effluent quality of wet coffee processing wastewater and its influence on downstream water quality. Ecohydrology and Hydrobiology, 18(2), 201-211. https://doi.org/10.1016/j. ecohyd.2017.10.007

Eaton, A. D., Clesceri, L. S., Rice, E. W., \& Greenberg, A. E. (Eds.). (2005). Standard Methods for the Examination of Water and Wastewater (21 $1^{\text {st }}$ ed.). Washington, DC, USA: APHA. ISBN-13: 978-0875530475.

González-Ávila, M. E., Vázquez-González, G. E., \& LópezBáez, W. (2020). Socioenvironmental affectation of coffee production activity in tributaries of La Suiza River at El Triunfo biosphere reserve, Chiapas. In A. Ortega-Rubio (Ed.) Socio-ecological studies in natural protected areas (pp. 381-403). Basingstoke, UK: Springer, Cham. https://doi. org/10.1007/978-3-030-47264-1_20

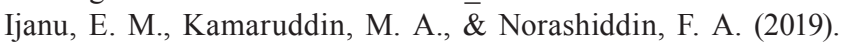 Coffee processing wastewater treatment: a critical review on current treatment technologies with a proposed alternative. Applied Water Science, 10, 11. https://doi.org/10.1007/s13201019-1091-9

INVEMAR (Instituto de Investigaciones Marinas y Costeras "José Benito Vives De Andréis") (Ed.). (2003). Manual de técnicas analiticas para la determinación de parámetros fisicoquímicos y contaminantes marinos (aguas, sedimentos y organismos). Consultado el 2 junio, 2020, desde http://www.invemar.org.co/redcostera1/invemar/ docs/7010manualTecnicasanaliticas.pdf

Kruskal, W. H., \& Wallis, W. A. (1952). Use of ranks in onecriterion variance analysis. Journal of the American Statistical Association, 47(260), 583-621.

Livingston, D. A. (1963). Chemical composition of Rivers and Lakes. In M. Fleischer (Ed.). Data of geochemistry (6th ed.). Washington, DC, USA: Government Printing Office.

López-Báez, W., Castro M., I., \& Camas G., R. (2014). Servicio ambiental de la reserva de la biósfera El Triunfo, Chiapas, México. Ingeniería Hidráulica y Ambiental, 35(3), 47-59. Consultado el 28 de mayo, 2021, desde http://scielo.sld.cu/ pdf/riha/v35n3/riha04314.pdf

López Castillo, D. (2012). Efecto del vertido directo de las aguas mieles en la calidad físico-química del agua de la Subcuenca del Río Jigüina, Jinotega. Revista Cientifica-FAREM Esteli/ Ciencias Ambientales, 1(1), 5-16.

Louzada A., L., Fonseca Andrade, L., \& de Oliveira, J. A. (2016). Evaluation of the toxic potential of coffee wastewater on seeds, roots and meristematic cells of Lactuca sativa L. Ecotoxicology and Environmental Safety, 133, 366-372. https://doi.org/10.1016/j.ecoenv.2016.07.019
Luthy, R. G. (1978). Manual of methods: preservation and analysis of coal gasification wastewaters. Pennsylvania, USA. Disponible en: https://www.osti.gov/servlets/purl/6366218. https://doi.org/10.2172/6366218

Matuk-Velázco, V., Puerta, G. I., \& Rodríguez, N. (1997). El impacto biológico de los efluentes del beneficio húmedo del café. Revista Cenicafé, 48(4), 234-252. Consultado el 12 de septiembre, 2020, desde https://www.researchgate.net/profile/ Gloria_Puerta-Quintero/publication/277116834

Medina-Meléndez, J. A., Ruiz-Nájera, R. E., Gómez-Castañeda, J. C., Sánchez-Yáñez, J. M., Gómez-Alfaro, G., \& Pinto-Molina, O. (2016). Estudio del sistema de producción de café (Coffea arabica L.) en la región Frailesca, Chiapas. Ciencia UAT, 10(2): 33-43.

Minuta, T., \& Jini, D. (2017). Impact of effluents from wet coffee processing plants on the Walleme river of Southern Ethiopia. Research Journal of Environmental Toxicology, 11(3), 90-96. https://doi.rg/10.3923/rjet.2017.90.96

NOM-001-ECOL-1996 (Norma Oficial Mexicana). (1996). Que establece los límites máximos permisibles de contaminantes en las descargas de aguas residuales en aguas y bienes nacionales. Diario Oficial de la Federación. D. F.: SEGOB. Consultado el 17 de octubre, 2018, desde http://dof.gob.mx/nota_detalle. php? codigo $=4863829 \&$ fecha $=06 / 01 / 1997$

NOM-127-SSA1-1994 (Norma Oficial Mexicana). (1994). Salud Ambiental. Agua para uso y consumo humano. Límites permisibles de calidad y tratamientos a que debe someterse el agua para su potabilización. Diario Oficial de la Federación. D. F.: SEGOB. Consultado el 23 de noviembre, 2018, desde https://agua.org.mx/wp-content/uploads/2016/10/nom127_ modificacion_2000.pdf

NOM-011-CONAGUA-2015 (Norma Oficial Mexicana). (2015). Conservación del recurso agua que establece las especificaciones y el método para determinar la disponibilidad media anual de las aguas nacionales. Diario Oficial de la Federación. D. F.: SEGOB. Consultado el 24 de noviembre, 2017, desde https://www.dof.gob.mx/nota_detalle. php? codigo $=5387027 \&$ fecha $=27 / 03 / 2015$

NMX-AA-029-SCFI-2001(Norma Mexicana). (2001). Análisis de aguas - determinación de fósforo total en aguas naturales, residuales y residuales tratadas - método de prueba. Secretaría de Economía Diario Oficial de la Federación. D. F.: SEGOB. Consultado el 24 de noviembre, 2018, desde https://www. gob.mx/cms/uploads/attachment/file/166773/NMX-AA-029SCFI-2001.pdf

NMX-AA-051-SCFI-2016 (Norma Mexicana). (2016). Análisis de agua medición de metales por absorción atómica en aguas naturales, potables, residuales y residuales tratadas método de prueba. Secretaria de Economía. Diario Oficial de la Federación. D. F.: SEGOB. Consultado el 24 de noviembre, 2018, desde https://http://www.economia-nmx.gob.mx/ normas/nmx/2010/nmx-aa-051-scfi-2016.pdf.

Patil, P. N., Sawant, D. V., \& Deshmukh, R. N. (2012). Physicochemical parameters for testing of water - A review. International Journal of Environmental Sciences, 3(3), 1194-1207.

Puerta Q., G. I. (2012). Factores, procesos y controles en la fermentación del café. Avances Técnicos Cenicafe, 422, 1-12. 
Rajendran, A., \& Mansiya, C. (2015). Physico-chemical analysis of ground water samples of coastal areas of south Chennai in the post-Tsunami scenario. Ecotoxicology and Environmental Safety, 121, 218-222. https://doi.org/10.1016/j. ecoenv.2015.03.037

Rigueira, R. J. de A., Lacerda Filho, A. F. de, Matos, A. T., Donzeles, S. M. L., \& Palacin, J. J. F. 2010. Alteração nas características físicas, químicas e bioquímicas da água no processo de lavagem, despolpa e desmucilagem de frutos do cafeeiro. Revista Engenharia Na Agricultura - Reveng, 18(2), 131-139. https://doi.org/10.13083/reveng.v18i2.214

Rodier, J., Legube, B., \& Merlet, N. (2011). Análisis de las aguas (9a ed.). Barcelona, España: Ediciones Omega. ISBN: 978-84282-1530-5.

SEDUE (Secretaría de Desarrollo Urbano y Ecología). (1989). ACUERDO por el que se establecen los Criterios Ecológicos de Calidad del Agua CE.CCA001/89. Diario Oficial de la Federación. D. F.: SEGOB. Consultado el 29 de junio, 2019, desde http://www.dof.gob.mx/nota_detalle. php? codigo $=4837548 \&$ fecha $=13 / 12 / 1989$

SEMARNAT-CONAGUA (Secretaría del Medio Ambiente y Recursos Naturales - Comisión Nacional del Agua). (1996). Normas Oficiales Mexicanas. NOM-001-SEMARNAT-1996. Consultado el 15 de enero, 2021, desde. http://www.conagua. gob.mx/CONAGUA07/Publicaciones/Publicaciones/SGAA15-13.pdf

SEMARNAT-CONAGUA (Secretaría del Medio Ambiente y Recursos Naturales - Comisión Nacional del Agua). (2019). Ley Federal de Derechos Disposiciones Aplicables en Materia de Aguas Nacionales 2019. Consultado el 28 junio, 2019, desde https://files.conagua.gob.mx/conagua/publicaciones/ Publicaciones/CGRF-1-19\%20LFD.pdf

SIAP (Servicio de Información Agroalimentaria y Pesquera). (2019). Anuario estadístico de la producción agrícola. Consultado el 20 de noviembre, 2020, desde https://nube.siap. gob.mx/cierreagricola/

SIAP (Servicio de Información Agroalimentaria y Pesquera). (2020). Avances de siembra y cosecha. Resumen por cultivo. Consultado el 04 de noviembre, 2020, desde http://infosiap. siap.gob.mx:8080/agricola_siap_gobmx/ResumenDelegacion.do
Tekle, D. Y., Hailu, A. B., Wassie, T. A., \& Tesema, A. G. (2015). Effect of coffee processing plant effluent on the physicochemical properties of receiving water bodies, jimma zone Ethiopia. American Journal of Environmental Protection, 4(2), 83-90. https://doi.org/10.11648/j.ajep.20150402.12

Padmapriya, R., Tharian, J. A., \& Thirunalasundari, T. (2013). Coffee waste management-An overview. International Journal of Current Research, 9(1), 83-91.

Tsigereda, K. T., Metadel, A. M., Mekibib, D. D., \& Fisseha, I. (2013). Effectiveness of advanced vs conventional wet coffee processing technologies in effluent wastewater quality. International Journal of Scientific and Engineering Research, 4(8), 1236-1243.

Torres-Valenzuela, L. S., Sanín-Villarrea, A., Arango-Ramírez, A., \& Serna-Jiménez, J. A. (2019). Caracterización fisicoquímica y microbiológica de aguas mieles del beneficio del café. Revista Ion, 32(2), 59-66. http://dx.doi.org/10.18273/revion. v32n2-2019006

US-EPA (United States-Environmental Protection Agency). (1986). Quality criteria for water, 1986. (EPA 440/5-86-001). Washington, DC, USA: Government Printing Office.

US-EPA (United States-Environmental Protection Agency). (2008). Onsite wastewater treatment system. Fact Sheet 9, Enhanced Nitrogen Removal-Nitrogen. Washington, DC, USA: Government Printing Office.

USDA (United States Department of Agriculture). (2020). Coffee: World markets and trade. Consultado el 18 de julio, 2020 desde https://apps.fas.usda.gov/psdonline/circulars/coffee.pdf

Woldesenbet, A. G., Woldeyes, B., Singh, B., \& Chandravanshi, B. S. (2014). Characteristics of wet coffee processing waste and its environmental impact in Ethiopia. International Journal of Research in Engineering and Science, 2(4), 1-5.

Woldesenbet, A. G., Woldeyes, B., \& Chandravanshi, B. S. (2015). Wet coffee processing waste management practice in Ethiopia. Asian Journal of Science and Technology, 6(05), 1467 -1471.

WHO (World Health Organization). (1995). Guideline for discharge of industrial effluent characteristics, 3 (pp. 231-236). Geneva, Switzerland: World Health Organization. 